Journal Club

Editor's Note: These short, critical reviews of recent papers in the Journal, written exclusively by graduate students or postdoctoral fellows, are intended to summarize the important findings of the paper and provide additional insight and commentary. For more information on the format and purpose of the Journal Club, please see http://www.jneurosci.org/misc/ifa_features.shtml.

\title{
When the Medial Prefrontal Cortex Fails: Implications for Extinction and Posttraumatic Stress Disorder Treatment
}

\author{
Peter A. Groblewski* and James M. Stafford* \\ Department of Behavioral Neuroscience, Oregon Health \& Science University, Portland, Oregon 97239 \\ Review of Kim et al.
}

Anxiety disorders such as posttraumatic stress disorder (PTSD) are characterized by a failure to inhibit maladaptive affective and visceral responses associated with environmental stimuli. One of the beststudied examples of this adaptive learning is fear extinction. In extinction, reexposure to a cue or context that was previously associated with a fear- or anxiety-provoking event results in the formation of a new inhibitory extinction memory between the cue and the outcome or response.

Current treatments for many anxiety disorders, including PTSD, capitalize on extinction or exposure-based therapies to decrease the powerful control that fearassociated environmental stimuli exert over behavior. Preclinical studies of various procedural factors (e.g., strength, frequency, and duration of extinction) and the neurobiological underpinnings of extinction memory formation are beginning to provide insight into ways to augment extinction-based therapies in the clinic (Myers and Davis, 2007). The timing of extinction training relative to initial

Received March 18, 2010; revised April 12, 2010; accepted April 16, 2010.

Preparation of this manuscript was supported by grants from the National Institutes of Health (AA018052, AA007702, and MH077111) and the Vertex Pharmaceutical Scholarship. We would also like to thank our advisors Christopher L. Cunningham and K. Matthew Lattal for their insightful comments.

*P.A.G. and J.M.S. contributed equally to this work.

Correspondence should be addressed to Peter A. Groblewski, Department of Behavioral Neuroscience, L470, Oregon Health \& Science University, 3181 SW Sam Jackson Park Road, Portland, OR 97239-3098. E-mail: groblews@ohsu.edu.

DOI:10.1523/JNEUROSCI.1413-10.2010

Copyright $\odot 2010$ the authors $\quad 0270-6474 / 10 / 307124-03 \$ 15.00 / 0$ memory acquisition is one such factor, and several studies have indicated that training administered immediately after acquisition results in less persistent suppression of the fear response than does extinction training after a longer delay, both in rodents and humans (e.g., Woods and Bouton, 2008; Huff et al., 2009).

It remains unknown, however, whether brain regions that control extinction, such as the medial prefrontal cortex (mPFC), are involved in the immediate extinction deficit (IED). The mPFC includes two subregions that are thought to exert opposing effects on fear: the prelimbic subregion (PL) is thought to mediate extinction failure by maintaining fear responses, while the infralimbic subregion (IL) is hypothesized to control decrements in fear response and strengthen extinction memory formation (Quirk and Mueller, 2008). Given the involvement of the mPFC in both expression and extinction of fear, an interesting possibility is that a failure to properly engage the mPFC causes the IED.

A recent The Journal of Neuroscience report by Kim et al. (2010) addressed this possibility and provided evidence that failure to activate the mPFC underlies the IED. Consistent with recent studies, this paper showed that extinction training conducted 15 min after fear acquisition (immediate group) failed to decrease fear responses on subsequent retention tests (i.e., it produced the IED), whereas extinction training conducted $24 \mathrm{~h}$ after fear acquisition (delayed group) resulted in robust retention of the extinction mem- ory. At the molecular level, Fos protein expression within IL and PL subregions of the $\mathrm{MPFC}$ was significantly elevated in the delayed group, but not in the immediate group. The increased cortical activation was specific to the $\mathrm{mPFC}$, as analysis of a negative-control region, the secondary motor cortex, showed no differences.

To confirm a causal relationship between the mPFC and the IED, Kim et al. (2010) artificially activated the IL during extinction training with implanted bipolar stimulating electrodes. This stimulation counteracted the IED seen in the immediate group, that is, the immediate group showed reduced levels of freezing similar to the delayed group during the posttraining, extinction-retrieval test.

By analyzing the IED at the behavioral, neural systems, and molecular levels, Kim et al. (2010) have provided powerful evidence that the neurobiological correlate of the IED is a lack of activation in the mPFC. These data raise many important basic and clinical issues, two of which will serve as the focus of this discussion. The first issue concerns the involvement of both the IL and PL subregions in the delay-sensitive components of extinction. The second issue involves the implications of these behavioral and neurobiological findings for treatment of PTSD and other associative disorders.

Dissociable involvement of the IL and PL subregions in extinction processes Using a combination of behavior, immunohistochemistry (IHC), and site-specific 
stimulation, Kim et al. (2010) identified the $\mathrm{mPFC}$ as a mediator of delay-sensitive extinction. It is particularly interesting that this analysis found increased Fos expression in both the IL and the PL following delayed extinction training, given the opposing roles of these subregions in extinction learning and fear expression (Quirk and Mueller, 2008). It is important to note, however, that Fos expression has limited temporal resolution. Therefore, it is possible that PL activation occurs in the early phases of extinction training when conditioned fear responding is greatest, whereas IL activation increases as fear responses decrease. Because IHC processing occurred 90 min after extinction training, the exact time of PL and IL activation during extinction is unclear. Future studies using other imaging techniques with more sensitive temporal resolution may provide additional detail of the temporal dynamics of IL and PL activation during extinction training (e.g., Guzowski et al., 2005).

Another explanation for why activity was detected in both the IL and PL regions despite their hypothesized opposing roles in fear extinction is that the Fos expression observed in the PL reflects activation of a subset of PL neurons that actually engage extinction processes. In fact, a study by Miller and Marshall (2004) showed that cue-induced Fos expression in the PL reflected an increase in GABAergic PL interneuron activation accompanied by a decrease in CaMKII expression in PL efferents, suggesting a net decrease in PL output. Alternatively, the increase in Fos expression in the PL may represent a select group of efferents that stimulate inhibitory interneurons in the basal amygdala, instead of those that activate excitatory projection neurons. Stimulation of these inhibitory interneurons in the amygdala by the PL would in turn reduce fear expression and could account for the decreased freezing exhibited by the delayed group during extinction training (Ehrlich et al., 2009). In any case, the increased levels of Fos expression in IL and PL found by Kim et al. (2010) further support a role for both of these regions in extinction memory formation, an effect that is sensitive to postacquisition delays.

Another finding by Kim et al. (2010) was that although both mPFC subregions were involved in retention of extinction learning, their activation during the normal loss of responding that occurs in extinction training existed only in the delayed group. This suggests that at short postacquisition intervals, fear expression and loss of response occurred independently of the mPFC and may rely solely on subcortical circuitry. Alternatively, the involvement of the mPFC in immediate extinction training could be limited to cellular processes upstream of transcription (i.e., cytoplasmic or membrane) and therefore not detected with Fos. However, future studies are needed to further characterize the delay-sensitive involvement of the $\mathrm{mPFC}$, and its dissociable subregions, in the loss of responding during extinction as well as retention of extinction memories.

\section{A role for the $\mathrm{MPFC}$ in augmenting exposure-based therapies}

The findings of Kim et al. (2010) are particularly relevant when considering the unfavorable outcomes of current treatments for PTSD. As the authors discuss, the current data on the impact of treatment delay on rehabilitation in the clinic are mixed and remain a contentious issue. However, the discrepant findings of these clinical studies are not surprising when considering the sensitivity of the IED phenomenon to procedural manipulations in preclinical animal models (Maren and Chang, 2006). Regardless, it is clear that in certain cases, extinction of fear involves a temporal component that can influence the outcome of extinction learning. Understanding the neurobiological underpinnings of these temporal characteristics will provide clinicians with more effective treatments, and may also have important implications for treatments of other disorders, including drug addiction, a disorder that frequently cooccurs with PTSD.

Patients with both PTSD and substance-use disorders (SUDs) are an especially relevant group as they typically exhibit a more severe clinical profile. In fact, it is thought that SUDs actually impair treatment of PTSD (and vice versa), because patients suffering from the two disorders are less responsive to treatment and exhibit higher rates of relapse (for review, see Schäfer and Najavits, 2007). Interestingly, a recent review synthesized preclinical and clinical data that strongly implicated the IL and PL regions of the mPFC as key modulators of expression and extinction of both conditioned fear and drug-seeking behavior (Peters et al., 2009). If a shared prefrontal cortex pathology underlies both PTSD and SUDs, it is possible that a single treatment regimen targeting this region could have positive outcomes for both disorders.

Early treatment of PTSD patients is important because delaying treatment could result in unnecessary exposure to stressors that might trigger drug craving and subsequent drug-seeking behavior. Resorting to these behaviors could further strengthen a patient's maladaptive associations that underlie PTSD and SUDs, thereby worsening symptoms and potentially impairing future treatment. One exciting possibility is that interventions that enhance the function of mPFC circuits could lead to even greater and more persistent decrements in fear and drugseeking behavior than exposure-based therapies alone. Several clinical studies have shown that stimulating the dorsolateral prefrontal cortex (human homolog of IL) reduced symptoms of PTSD with no adverse behavior or emotional effects (Boggio et al., 2009). Therefore, future combinations of similar mPFC-targeted manipulations with timely behavioral intervention may eliminate potential IEDs in a manner consistent with the findings of Kim et al. (2010), thereby reducing unnecessary exposure to traumatic stressors when treatment is delayed. It is important to note, however, that relapse frequently occurs after exposure-based therapies in the clinic and after extinction in animals. Therefore, although Kim et al. (2010) did not report findings of long-term retention of extinction (e.g., spontaneous recovery or reinstatement), future studies are necessary to assess the effect of mPFC manipulations on the persistence of immediate- and delayed-extinction training. These findings are especially important when considering treatment interventions of patients with PTSD and SUDs.

In conclusion, the results of Kim et al. (2010) are some of the first to identify the neurobiological mechanisms underlying the effects of delay intervals on extinction of conditioned fear in rodents. These data identified the mPFC as a delay-sensitive modulator of extinction memory formation and provide a promising target for future studies aimed at identifying the specific neurobiological underpinnings of the IED phenomenon. By furthering our understanding of the temporal and neurobiological characteristics of extinction of conditioned fear, these studies will help to develop more effective behavioral and pharmacological treatments of PTSD and other disorders such as drug addiction.

\section{References}

Boggio PS, Rocha M, Oliveira MO, Fecteau S, Cohen RB, Campanha C, Ferreira-Santos E, Meleiro A, Corchs F, Zaghi S, Pascual-Leone A, Fregni F (2009) Noninvasive brain stimulation with high-frequency and low-intensity repetitive transcranial magnetic stimulation 
treatment for posttraumatic stress disorder. J Clin Psychiatry. Advance online publication. Retrieved March 8, 2010. doi:10.4088/JCP.08m04638blu.

Ehrlich I, Humeau Y, Grenier F, Ciocchi S, Herry C, Lüthi A (2009) Amygdala inhibitory circuits and the control of fear memory. Neuron 62:757-771.

Guzowski JF, Timlin JA, Roysam B, McNaughton BL, Worley PF, Barnes CA (2005) Mapping behaviorally relevant neural circuits with immediate-early gene expression. Curr Opin Neurobiol 15:599-606.

Huff NC, Hernandez JA, Blanding NQ, LaBar KS (2009) Delayed extinction attenuates conditioned fear renewal and spontaneous recovery in humans. Behav Neurosci 123: $834-843$.

Kim SC, Jo YS, Kim IH, Kim H, Choi JS (2010) Lack of medial prefrontal cortex activation underlies the immediate extinction deficit. J Neurosci 30:832-837.

Maren S, Chang CH (2006) Recent fear is resistant to extinction. Proc Natl Acad Sci U S A 103:18020-18025.

Miller CA, Marshall JF (2004) Altered prelimbic cortex output during cue-elicited drug seeking. J Neurosci 24:6889-6897.

Myers KM, Davis M (2007) Mechanisms of fear extinction. Mol Psychiatry 12:120-150.
Peters J, Kalivas PW, Quirk GJ (2009) Extinction circuits for fear and addiction overlap in prefrontal cortex. Learn Mem 16:279-288.

Quirk GJ, Mueller D (2008) Neural mechanisms of extinction learning and retrieval. Neuropsychopharmacology 33:56-72.

Schäfer I, Najavits LM (2007) Clinical challenges in the treatment of patients with posttraumatic stress disorder and substance abuse. Curr Opin Psychiatry 20:614-618.

Woods AM, Bouton ME (2008) Immediate extinction causes a less durable loss of performance than delayed extinction following either fear or appetitive conditioning. Learn Mem 15:909-920. 